\title{
Number of Sylow subgroups in finite groups
}

\author{
Wenbin Guo and Evgeny P. Vdovin \\ Communicated by Evgenii I. Khukhro
}

\begin{abstract}
Denote by $v_{p}(G)$ the number of Sylow $p$-subgroups of $G$. It is not difficult to see that $v_{p}(H) \leqslant v_{p}(G)$ for $H \leqslant G$, however $v_{p}(H)$ does not divide $v_{p}(G)$ in general. In this paper we reduce the question whether $v_{p}(H)$ divides $v_{p}(G)$ for every $H \leqslant G$ to almost simple groups. This result substantially generalizes the previous result by G. Navarro and also provides an alternative proof of Navarro's theorem.
\end{abstract}

\section{Introduction}

Throughout this paper, all groups are finite. Let $G$ be a group and $p$ a prime. Denote by $v_{p}(G)$ the number of Sylow $p$-subgroups of $G$. It is a trivial exercise to check that $v_{p}(H) \leqslant v_{p}(G)$ for every subgroup $H$ of $G$. However $v_{p}(H)$ does not necessarily divide $\nu_{p}(G)$ in general. For example, let $G=A_{5}$ and $H=A_{4}$, then $\nu_{3}(G)=10$ and $\nu_{3}(H)=4$. In 2003, G. Navarro [6] proved that if $G$ is $p$-solvable, then $v_{p}(H)$ divides $v_{p}(G)$ for every $H \leqslant G$.

We say that a group $G$ satisfies $\operatorname{DivSyl}(p)$ if $v_{p}(H)$ divides $v_{p}(G)$ for every $H \leqslant G$. Our goal is to prove that $G$ satisfies $\operatorname{DivSyl}(p)$ provided every nonabelian composition factor of $G$ satisfies DivSyl( $p$ ) (see Theorem 1.1 below). This result substantially generalizes the result by Navarro since in $p$-solvable groups all nonabelian composition factors evidently satisfy $\operatorname{DivSyl}(p)$. Our technique can also be applied to derive Navarro's theorem, so we provide an alternative proof for Navarro's theorem.

In order to formulate the main theorem, we need to recall some definitions.

Let $A, B, H$ be subgroups of $G$ such that $B \Vdash A$. Define the normalizer $N_{H}(A / B)$ of $A / B$ in $H$ by $N_{H}(A) \cap N_{H}(B)$. If $x \in N_{H}(A / B)$, then $x$ induces an automorphism of $A / B$ acting by $B a \mapsto B x^{-1} a x$. Thus there exists a homomorphism $N_{H}(A / B) \rightarrow \operatorname{Aut}(A / B)$. The image of the homomorphism is denoted by $\operatorname{Aut}_{H}(A / B)$ and is called the group of $H$-induced automorphisms on $A / B$,

The first author is supported by a NNSF grant of China (grant \#11771409) and the Wu WenTsun Key Laboratory of Mathematics of the Chinese Academy of Sciences. The second author is supported by Chinese Academy of Sciences President's International Fellowship Initiative (grant \#2017VMA0049). 
while the kernel of the homomorphism is denoted by $C_{H}(A / B)$. If $B=1$, then we simply write $\operatorname{Aut}_{H}(A)$. The groups of induced automorphisms were introduced by F. Gross in [1], where the author says that this notion is taken from unpublished lectures by $\mathrm{H}$. Wielandt. Clearly $C_{H}(A / B)=C_{G}(A / B) \cap H$, so

$$
\begin{aligned}
\operatorname{Aut}_{H}(A / B) & =N_{H}(A / B) / C_{H}(A / B) \\
& \simeq N_{H}(A / B) C_{G}(A / B) / C_{G}(A / B) \leqslant \operatorname{Aut}_{G}(A / B),
\end{aligned}
$$

that is, $\operatorname{Aut}_{H}(A / B)$ can be embedded into $\operatorname{Aut}_{G}(A / B)$ in a natural way. Hence, without loss of generality, we may assume that $\operatorname{Aut}_{H}(A / B)$ is a subgroup of $\operatorname{Aut}_{G}(A / B)$.

A composition series is called an $(r c)$-series if it is a refinement of a chief series. (This notion was introduced by V. A. Vedernikov in [11].)

Theorem 1.1 (Main theorem). Let

$$
1=G_{0}<G_{1}<\cdots<G_{n}=G
$$

be an ( $r c$ )-series of $G$. Assume that, for each nonabelian $G_{i} / G_{i-1}$ and for every p-subgroup $P$ of $\operatorname{Aut}_{G}\left(G_{i} / G_{i-1}\right)$, the group $P\left(G_{i} / G_{i-1}\right)$ satisfies DivSyl $(p)$. Then $G$ satisfies $\operatorname{DivSyl}(p)$.

Notice that if $G_{i} / G_{i-1}$ (in the notation of the theorem) is nonabelian simple, it has a natural embedding into $\operatorname{Aut}_{G}\left(G_{i} / G_{i-1}\right)$ as a group of its inner automorphisms, and we identify $G_{i} / G_{i-1}$ with a normal subgroup of $\operatorname{Aut}_{G}\left(G_{i} / G_{i-1}\right)$ of all inner automorphisms.

At the end of the paper, we discuss possible ways of extending this statement and also explain how Navarro's result can be derived from this theorem.

\section{Preliminaries}

We write $H \leqslant G$ and $H \preccurlyeq G$ if $H$ is a subgroup of $G$ and $H$ is a normal subgroup of $G$, respectively, and $p$ denotes a prime number. A natural number $n$ is called a $p$-number (respectively a $p^{\prime}$-number) if it is a power of $p$ (respectively if it is coprime to $p$ ). We denote by $n_{p}$ and $n_{p^{\prime}}$ the $p$ - and $p^{\prime}$-part of the natural number $n$. A group is said to be a $p$-group (respectively a $p^{\prime}$-group) if its order is a $p$-number (respectively a $p^{\prime}$-number). A group $G$ is called $p$-solvable if it possesses a subnormal series with all sections either $p$ - or $p^{\prime}$-groups. If $\varphi: X \rightarrow Y$ is a map, then $\varphi(x)$ denotes the image of $x \in X$, while for $\sigma \in \operatorname{Sym}_{k}$ we write $i \sigma$ for the image of $i \in\{1, \ldots, k\}$ under $\sigma$. By $\operatorname{Syl}_{p}(G)$ we denote the set of all 
Sylow $p$-subgroups of $G$. All unexplained notation and definitions can be found in [4].

The following elementary result follows from [2, Theorem 2.1].

Lemma 2.1. Assume that $A \lessgtr G$ and $P \in \operatorname{Syl}_{p}(G)$. Then

$$
v_{p}(G)=v_{p}(G / A) \cdot v_{p}(P A)
$$

for some (hence for every) $P \in \operatorname{Syl}_{p}(G)$, and $v_{p}(P A)=\left|A: N_{A}(P)\right|$.

Lemma 2.2. Assume that $A \preccurlyeq G$ and both $A P$ and $G / A$ satisfy DivSyl $(p)$. Then $G$ satisfies $\operatorname{DivSyl}(p)$.

Proof. Let $H \leqslant G$. Choose $Q \in \operatorname{Syl}_{p}(G)$ so that $Q \cap H \in \operatorname{Syl}_{p}(H)$. Since $P A$ and $Q A$ are conjugate, $Q A$ satisfies $\operatorname{DivSyl}(p)$. Now by Lemma 2.1,

$$
v_{p}(G)=v_{p}(G / A) \cdot v_{p}(Q A),
$$

and

$$
\begin{aligned}
v_{p}(H) & =v_{p}(H /(H \cap A)) \cdot v_{p}((Q \cap H)(H \cap A)) \\
& =v_{p}(H A / A) \cdot v_{p}((Q \cap H)(H \cap A)) .
\end{aligned}
$$

Since $H A / A \leqslant G / A$ and $(Q \cap H)(H \cap A) \leqslant Q A$, the conditions of the lemma imply $v_{p}(H A / A)$ divides $v_{p}(G / A)$ and $v_{p}((Q \cap H)(H \cap A))$ divides $v_{p}(Q A)$. This implies that $v_{p}(H)$ divides $v_{p}(G)$.

The following lemma is proven in [6], but now it can be easily derived from the above two lemmas and we provide here an alternative proof of it.

Lemma 2.3 ([6, Theorem A]). Let $H$ be a subgroup of a p-solvable group $G$. Then $v_{p}(H)$ divides $v_{p}(G)$.

Proof. We argue by induction on $|G|$. Let $A$ be a minimal normal subgroup of $G$ and $H \leqslant G$. Let $P \in \operatorname{Syl}_{p}(G)$ with $P \cap H \leqslant \operatorname{Syl}_{p}(H)$. By Lemma 2.1, we have

$$
v_{p}(G)=v_{p}(G / A) \cdot v_{p}(P A)
$$

and

$$
v_{p}(H)=v_{p}(H A / A) \cdot v_{p}((H \cap P)(H \cap A)) .
$$

By induction, $v_{p}(H A / A)$ divides $v_{p}(G / A)$. Since $G$ is $p$-solvable, $A$ is either a $p$-group or a $p^{\prime}$-group. 
If $A$ is a $p$-group, then $v_{p}((H \cap P)(H \cap A))=1$, so $v_{p}(H)$ divides $v_{p}(G)$.

If $A$ is a $p^{\prime}$-group, then $|A|$ and $|P|$ are coprime, in particular $P \cap A=1$. Since $P$ normalizes $A$, we have that $\left[N_{A}(P), P\right] \leqslant A \cap P=1$, so $N_{A}(P)=C_{A}(P)$. The known properties of coprime action (see, for example, [4, Exercise 3E.4]) and Lemma 2.1 imply that $v_{p}((H \cap P)(H \cap A))=\left|(H \cap A): C_{H \cap A}(H \cap P)\right|$ divides $\left|A: C_{A}(H \cap P)\right|$, while $\left|A: C_{A}(H \cap P)\right|$ divides $\left|A: C_{A}(P)\right|=v_{p}(P A)$. Thus $v_{p}(H)$ divides $v_{p}(G)$.

Recall that a group $A$ is said to be an almost simple group if there exists a simple nonabelian group $S$ such that $S \leqslant A \leqslant \operatorname{Aut}(S)$. By the Schreier conjecture $\operatorname{Aut}(S) / S$ is solvable for each simple group $S$.

Corollary 2.4. Let $A$ be an almost simple group with simple socle $S$ and $P \in$ $\operatorname{Syl}_{p}(A)$. If PS satisfies $\operatorname{DivSyl}(p)$, then A satisfies $\operatorname{DivSyl}(p)$.

Proof. This follows from Lemmas 2.2 and 2.3 since $A / S$ is solvable.

Lemma 2.5 ([9, Theorem 1]). Let

$$
1=G_{0}<G_{1}<\cdots<G_{n}=G
$$

be an $(r c)$-series of $G$ and denote the section $G_{i} / G_{i-1}$ by $S_{i}$. Let

$$
1=H_{0}<H_{1}<\cdots<H_{n}=G
$$

be a composition series of $G$. Then there exist a permutation $\sigma \in \mathrm{Sym}_{n}$ such that $\operatorname{Aut}_{G}\left(H_{i} / H_{i-1}\right) \leqslant \operatorname{Aut}_{G}\left(S_{i \sigma}\right)$ for $i=1, \ldots, n$. Moreover, if the second series is an $(r c)$-series, then $\sigma$ can be chosen so that $\operatorname{Aut}_{G}\left(H_{i} / H_{i-1}\right) \simeq \operatorname{Aut}_{G}\left(S_{i \sigma}\right)$ for $i=1, \ldots, n$.

The following simple lemma plays a technical role.

Lemma 2.6. Let $G$ be a finite group, possessing a normal series

$$
1 \triangleleft H \triangleleft K \triangleleft G
$$

such that $H$ is a p-group, $K / H$ is a $p^{\prime}$-group and $G / K$ is a $p$-group. Let $Q$ be a $p$-subgroup of $G$ containing $H$ and let $P$ be a Sylow p-subgroup of $G$ containing $Q$. Consider a natural homomorphism

$$
\text { - }: G \rightarrow \bar{G}=G / H \text {. }
$$

Then $\overline{N_{K}(Q)}=N_{\bar{K}}(\bar{Q})=C_{\bar{K}}(\bar{Q})$ and $N_{K}(P) \leqslant N_{K}(Q)$. 
Proof. $\overline{N_{K}(Q)} \subseteq N_{\bar{K}}(\bar{Q})$ is evident. Consider $\bar{x} \in N_{\bar{K}}(\bar{Q})$. Then every inverse image $x$ of $\bar{x}$ normalizes the full inverse image of $\bar{Q}$. Since $H \leqslant Q$, the full inverse image of $\bar{Q}$ equals $Q$, so $x$ normalizes $Q$. Consequently, $N_{\bar{K}}(\bar{Q}) \subseteq \overline{N_{K}(Q)}$. By the conditions of the lemma, $\bar{K}=O_{p^{\prime}}(\bar{G})$, so $\bar{K} \leqslant \bar{G}$ and $|\bar{K}|$ and $|\bar{Q}|$ are coprime. Thus $N_{\bar{K}}(\bar{Q})=C_{\bar{K}}(\bar{Q})$. Since $H \leqslant P$ and $P$ is a $p$-group, the identity

$$
\overline{N_{K}(P)}=N_{\bar{K}}(\bar{P})=C_{\bar{K}}(\bar{P})
$$

holds. Clearly $C_{\bar{K}}(\bar{P}) \leqslant C_{\bar{K}}(\bar{Q})$. It follows that $N_{K}(P) \leqslant N_{K}(Q)$.

In this paper we often consider subgroups of a permutation wreath product, so we fix notation for such groups. If $L$ is a group and $K$ is a subgroup of the symmetric group $\operatorname{Sym}_{k}$, then $L \imath K$ denotes the permutation wreath product. By definition $L \prec K$ is isomorphic to a semidirect product $\left(L_{1} \times \cdots \times L_{k}\right) \rtimes K$, where $L_{1} \simeq \cdots \simeq L_{k} \simeq L$ and $K$ permutes the $L_{i}$ 's, that is, an arbitrary $\sigma \in K$ acts by

$$
\sigma:\left(g_{1}, \ldots, g_{k}\right) \mapsto\left(g_{1 \sigma^{-1}}, \ldots, g_{k \sigma^{-1}}\right)
$$

for $\left(g_{1}, \ldots, g_{k}\right) \in L_{1} \times \cdots \times L_{k}$. We always denote $L_{1} \times \cdots \times L_{k}$ by $\mathbf{L}$.

Suppose that $G$ is a subgroup of $L \imath K$. Let $\rho: G \rightarrow \operatorname{Sym}_{k}$ be the homomorphism corresponding to the action of $G$ on $\left\{L_{1}, \ldots, L_{k}\right\}$ by conjugation. Each element $g$ of $G$ as an element from $L \imath K$ can be uniquely written as

$$
g=\left(g_{1}, \ldots, g_{k}\right) \rho(g),
$$

where $g_{i} \in L_{i}$, and $\rho(g) \in \operatorname{Sym}_{k}$ permutes the $g_{i}$ 's. Given $g=\left(g_{1}, \ldots, g_{k}\right) \rho(g)$ of $L \imath K$, define $\pi_{i}(g)$ to be equal to $g_{i}$. In particular, the restriction of $\pi_{i}$ on $\mathbf{L}$ is the natural projection on $L_{i}$.

Now if $S$ is a normal subgroup of $L$, then we can construct a normal subgroup $\mathbf{S}=S_{1} \times \cdots \times S_{k}$ of $L \imath K$ such that

$$
S_{1} \simeq \cdots \simeq S_{k} \simeq S \text { and }(L \prec K) / \mathbf{S} \simeq(L / S) \prec K .
$$

In order to construct $\mathbf{S}$ we need to take one representative $L_{j}$ from every $K$-orbit on $\left\{L_{1}, \ldots, L_{k}\right\}$, then in each representative we need to take $S_{j} \preccurlyeq L_{j}$ such that $S_{j} \simeq S$ and $L_{j} / S_{j} \simeq L / S$, and finally define $\mathbf{S}$ to be equal to the normal closure of the subgroup generated by $S_{j}$ 's. From the construction it is evident that if $K$ is transitive, then $\mathbf{S}$ is uniquely defined by the choice of $S_{1} \leqslant L_{1}$.

Lemma 2.7. Assume that $T$ is a unique minimal normal subgroup of $G$. Assume also that $T$ is nonabelian, i.e. $T=S_{1} \times \cdots \times S_{k}$, where $S_{1}, \ldots, S_{k}$ are isomorphic (moreover, G-conjugate) nonabelian simple groups. Let $\rho: G \rightarrow \operatorname{Sym}_{k}$ be 
the permutation representation corresponding to the action of $G$ on $\left\{S_{1}, \ldots, S_{k}\right\}$ by conjugation. Then there exists an injective homomorphism

$$
\varphi: G \rightarrow \operatorname{Aut}_{G}\left(S_{1}\right) \curlywedge \rho(G)=\left(\operatorname{Aut}_{G}\left(S_{1}\right) \times \cdots \times \operatorname{Aut}_{G}\left(S_{k}\right)\right) \rtimes \rho(G) .
$$

Moreover, if $H \leqslant G$ and $H T=G$, then $\varphi$ can be chosen so that for every $H \leqslant$ $X \leqslant G$ we have

$$
\begin{aligned}
\varphi(X) & \leqslant \operatorname{Aut}_{X}\left(S_{1}\right) \imath \rho(X) \\
& =\operatorname{Aut}_{X}\left(S_{1}\right) \prec \rho(G)=\left(\operatorname{Aut}_{X}\left(S_{1}\right) \times \cdots \times \operatorname{Aut}_{X}\left(S_{k}\right)\right) \rtimes \rho(X) .
\end{aligned}
$$

Proof. The existence of $\varphi$ is known (see, for example, [10, Lemma 2]). Hence, we only need to show that $\varphi(X) \leqslant \operatorname{Aut}_{X}\left(S_{1}\right) \geq \rho(X)$. Here we provide the construction of $\varphi$ and leave all technical details for the reader.

First notice that $\rho(G)$ is transitive since $T$ is a minimal normal subgroup. By the fundamental counting principle (see [4, Theorem 1.4]), the action of $G$ on $\left\{S_{1}, \ldots, S_{k}\right\}$ is equivalent to the action of $G$ on right cosets of $N_{G}\left(S_{1}\right)$ by right multiplication. By [3, Theorem IV.1.4 a)], there exists an injective homomorphism

$$
\psi: G \rightarrow\left(N_{G}\left(S_{1}\right) \times \cdots \times N_{G}\left(S_{k}\right)\right) \rtimes \rho(G)=N_{G}\left(S_{1}\right) \prec \rho(G),
$$

defined in the following way. Fix right coset representatives $r_{1}, \ldots, r_{k}$ of $N_{G}\left(S_{1}\right)$ in $G$. Given $g \in G$, define the element $n_{i}(g)$ of $N_{G}\left(S_{1}\right)$ by

$$
r_{i} g=n_{i}(g) r_{i \rho(g)}
$$

Then $\psi$ maps $g$ to $\left(n_{1}(g), \ldots, n_{k}(g)\right) \rho(g)$.

Consider a normal subgroup $C_{G}\left(S_{1}\right)$ of $N_{G}\left(S_{1}\right)$. Since $\rho(G)$ is transitive, we have that $C_{G}\left(S_{1}\right) \times \cdots \times C_{G}\left(S_{k}\right)$ is a normal subgroup. Consider a natural homomorphism

$$
\theta: N_{G}\left(S_{1}\right) \prec \rho(G) \rightarrow\left(N_{G}\left(S_{1}\right) \prec \rho(G)\right) /\left(C_{G}\left(S_{1}\right) \times \cdots \times C_{G}\left(S_{k}\right)\right) .
$$

Since $T$ is the unique minimal normal subgroup of $G$ and $T$ is nonabelian, we have $\psi(G) \cap \operatorname{Ker}(\theta)=1$, while $\theta\left(N_{G}\left(S_{1}\right) \geq \rho(G)\right)=\operatorname{Aut}_{G}\left(S_{1}\right) \geq \rho(G)$ by definition.

Now assume that $H \leqslant G$ is chosen so that $H T=G$. Then the right coset representatives $r_{1}, \ldots, r_{k}$ of $N_{G}\left(S_{1}\right)$ can be chosen from $H$. Hence if $H \leqslant X \leqslant G$, then for every $x \in X$ and $i=1, \ldots, k$, we have

$$
n_{i}(x)=r_{i} x r_{i \rho(x)}^{-1} \in X \cap N_{G}\left(S_{1}\right)=N_{X}\left(S_{1}\right) .
$$

Therefore $\psi(X) \leqslant N_{X}\left(S_{1}\right) \prec \rho(X)$, and so

$$
\varphi(X)=\theta \psi(X) \leqslant \operatorname{Aut}_{X}\left(S_{1}\right) \imath \rho(X) .
$$


Lemma 2.8. Let $L$ be a group with a normal subgroup $S$ such that $L / S$ is a $p$ group. Let $K$ be a transitive $p$-subgroup of $\operatorname{Sym}_{k}$. Choose $S_{1} \leqslant L_{1}$ with $S_{1} \simeq S$ and $L_{1} / S_{1} \simeq L / S$, then $\mathbf{S}=S_{1}^{L \imath K}=S_{1} \times \cdots \times S_{k}$. Let $G$ be a subgroup of $L$ < $K$ satisfying the following conditions:

(a) $\mathbf{S} \leqslant G$;

(b) $G \mathbf{L}=L$ २ $K$;

(c) $\pi_{i}\left(N_{G}\left(S_{i}\right)\right)=L_{i}$;

(d) for some $Q \in \operatorname{Syl}_{p}(G)$, if $P_{i}=\pi_{i}\left(N_{Q}\left(S_{i}\right)\right)$, then $Q \leqslant\left(P_{1} \times \cdots \times P_{k}\right) \rtimes K$.

Then the restriction of $\pi_{i}$ on $N_{G}\left(S_{i}\right)$ is a homomorphism and

$$
v_{p}(G)=|S|_{p^{\prime}}^{k-1} \cdot v_{p}(L) .
$$

Proof. Since $G \mathbf{L}=L \nmid K$, we have $\rho(G)=G \mathbf{L} / \mathbf{L} \simeq G /(G \cap \mathbf{L}) \simeq K$, in particular, $G /(G \cap \mathbf{L})$ is a $p$-group. Hence $G=Q(G \cap \mathbf{L})$ and $L \imath K=Q \mathbf{L}$, in particular $\rho(Q)=K$. Now $g=\left(g_{1}, \ldots, g_{k}\right) \rho(g) \in N_{L \backslash K}\left(S_{i}\right)$ if and only if $i \rho(g)=i$. For every $g, h \in L \imath K$ we have $\pi_{i}(g) \cdot \pi_{i}(h)=g_{i} \cdot h_{i}$. On the other hand, if we take $g, h \in N_{L \backslash K}\left(S_{i}\right)$, then

$$
\begin{aligned}
\pi_{i}(g h) & =\pi_{i}\left(\left(g_{1}, \ldots, g_{k}\right) \rho(g) \cdot\left(h_{1}, \ldots, h_{k}\right) \rho(h)\right) \\
& =\pi_{i}\left(\left(g_{1}, \ldots, g_{k}\right) \cdot\left(h_{1}, \ldots, h_{k}\right)^{\rho(g)^{-1}} \cdot \rho(g) \rho(h)\right) \\
& =\pi_{i}\left(\left(g_{1}, \ldots, g_{k}\right) \cdot\left(h_{1 \rho(g)}, \ldots, h_{i}, \ldots, h_{k \rho(g)}\right) \cdot \rho(g) \rho(h)\right) \\
& =\pi_{i}\left(\left(g_{1} \cdot h_{1 \rho(g)}, \ldots, g_{i} \cdot h_{i}, \ldots, g_{k} \cdot h_{k \rho(g)}\right) \cdot \rho(g) \rho(h)\right)=g_{i} \cdot h_{i},
\end{aligned}
$$

thus the restriction of $\pi_{i}$ on $N_{G}\left(S_{i}\right)$ is a homomorphism.

Since $L / S$ is a $p$-group and $K$ is a $p$-group, we have that $(L<K) / \mathbf{S}$ is a $p$ group. Hence $G / \mathbf{S}$ is a $p$-group and $Q \mathbf{S}=G$. Denote $\pi_{i}(Q \cap \mathbf{S})$ by $Q_{i}$. Clearly $Q \cap S_{i} \leqslant Q_{i}$. On the other hand, since $S_{i}$ is subnormal in $G, Q \cap S_{i}$ is a Sylow $p$-subgroup of $S_{i}$, so $Q \cap S_{i}=Q_{i}$. Since $\mathbf{S}$ normalizes $S_{i}$ and $G=Q \mathbf{S}$, we have $N_{G}\left(S_{i}\right)=N_{Q}\left(S_{i}\right) \mathbf{S}$, therefore $\pi_{i}\left(N_{G}\left(S_{i}\right)\right)=P_{i} S_{i}=L_{i}$. But since

$$
\begin{aligned}
\left|P_{i}\right| & =\left|P_{i} /\left(P_{i} \cap S_{i}\right)\right| \cdot\left|P_{i} \cap S_{i}\right| \\
& =\left|P_{i} /\left(P_{i} \cap S_{i}\right)\right| \cdot\left|\pi_{i}\left(N_{Q}\left(S_{i}\right)\right) \cap S_{i}\right| \\
& =\left|P_{i} /\left(P_{i} \cap S_{i}\right)\right| \cdot\left|\pi_{i}\left(N_{Q}\left(S_{i}\right) \cap S_{i}\right)\right| \\
& =\left|P_{i} /\left(P_{i} \cap S_{i}\right)\right| \cdot\left|\pi_{i}\left(Q \cap S_{i}\right)\right| \\
& =\left|L_{i} / S_{i}\right| \cdot\left|Q_{i}\right|=\left|L_{i}\right|_{p},
\end{aligned}
$$


we obtain $P_{i} \in \operatorname{Syl}_{p}\left(L_{i}\right)$. Moreover, since $Q$ as a subgroup of $G$ permutes $S_{i}$ 's, we have that $Q$ permutes $N_{Q}\left(S_{i}\right)$ 's, and so $Q$ permutes $P_{i}$ 's. This implies that $Q$ normalizes $P_{1} \times \cdots \times P_{k}$. Consequently, $\rho(Q)=K$ normalizes $P_{1} \times \cdots \times P_{k}$ and the subgroup $P=\left(P_{1} \times \cdots \times P_{k}\right) \rtimes K$ in condition (d) of the lemma is correctly defined. Since $P_{i} \in \operatorname{Syl}_{p}\left(L_{i}\right)$ and $K$ is a $p$-group, we also have that $P \in$ $\operatorname{Syl}_{p}(L<S)$. Then by condition (d), $Q \leqslant P$, and so $Q=P \cap G$.

Since $G / S$ is a $p$-group, Lemma 2.1 implies that

$$
v_{p}(G)=\left|\mathbf{S}: N_{\mathbf{S}}(Q)\right|
$$

so

$$
v_{p}(G)=\frac{|\mathbf{S}|_{p^{\prime}}}{\left|N_{\mathbf{S}}(Q)\right|_{p^{\prime}}} .
$$

Since $L_{1}=P_{1} S_{1}$, by Lemma 2.1 again, we have

$$
v_{p}\left(L_{1}\right)=\left|S_{1}: N_{S_{1}}\left(P_{1}\right)\right|=\frac{\left|S_{1}\right|_{p^{\prime}}}{\left|N_{S_{1}}\left(P_{1}\right)\right|_{p^{\prime}}} .
$$

If we can show that

$$
\left|N_{\mathbf{S}}(Q)\right|_{p^{\prime}}=\left|N_{S_{1}}\left(P_{1}\right)\right|_{p^{\prime}},
$$

then (2.1) and (2.2) would imply

$$
v_{p}(G)=\frac{|\mathbf{S}|_{p^{\prime}}}{\left|N_{\mathbf{S}}(Q)\right|_{p^{\prime}}}=\frac{|\mathbf{S}|_{p^{\prime}}}{\left|S_{1}\right|_{p^{\prime}}} \cdot \frac{\left|S_{1}\right|_{p^{\prime}}}{\left|N_{S_{1}}\left(P_{1}\right)\right|_{p^{\prime}}}=\left|S_{1}\right|_{p^{\prime}}^{k-1} \cdot v_{p}\left(L_{1}\right)
$$

and so the lemma follows.

Thus we only have to prove (2.2).

Denote $N_{S_{i}}\left(P_{i}\right)$ by $X_{i}$ and let $\mathbf{X}=X_{1} \times \cdots \times X_{k}$. Clearly

$$
Q_{i}=P_{i} \cap S_{i} \preccurlyeq X_{i},
$$

and since $Q_{i}$ is a Sylow $p$-subgroup of $S_{i}, Q_{i}$ is a (normal) Sylow $p$-subgroup of $X_{i}$. Therefore $Q_{1} \times \cdots \times Q_{k}=O_{p}(\mathbf{X}) \in \operatorname{Syl}_{p}(\mathbf{X})$ and $\mathbf{X}$ is an extension of a $p$-group by a $p^{\prime}$-group. We show that $P$ normalizes $\mathbf{X}$ and that $N_{\mathbf{S}}(Q)$ lies in $\mathbf{X}$. By construction, $P$ permutes $P_{i}$ 's, so $P$ permutes $X_{i}$ 's, therefore $P$ normalizes $X_{1} \times \cdots \times X_{k}=\mathbf{X}$. In particular, $\rho(P)=\rho(Q)=K$ normalizes $\mathbf{X}$ and $\mathbf{X} K=X_{1} \prec K$. In order to show that $N_{\mathbf{S}}(Q) \leqslant \mathbf{X}$, consider $x=\left(x_{1}, \ldots, x_{k}\right) \in$ $N_{\mathbf{S}}(Q)$. Since $x$ normalizes $Q$ and $S_{i}$, we obtain that $x$ normalizes $N_{Q}\left(S_{i}\right)$ for $i=1, \ldots, k$. Take $y=\left(y_{1}, \ldots, y_{k}\right) \rho(y) \in N_{Q}\left(S_{i}\right)$. Then $i \rho(y)=i$. Recall that 
by condition (d) of the lemma $Q \leqslant P$, so $y_{i} \in P_{i}$ for $i=1, \ldots, k$. Since $x_{j}$ centralizes $y_{t}$ for $j \neq t$, we obtain

$$
\begin{aligned}
y^{x}= & \left(y_{1}^{x_{1}}, \ldots, y_{k}^{x_{k}}\right) \cdot \rho(y)^{x} \\
= & \left(y_{1}^{x_{1}}, \ldots, y_{k}^{x_{k}}\right) \cdot\left(x_{1}^{-1}, \ldots, x_{k}^{-1}\right) \cdot\left(x_{1}, \ldots, x_{k}\right)^{\rho(y)^{-1}} \cdot \rho(y) \\
= & \left(y_{1}^{x_{1}}, \ldots, y_{k}^{x_{k}}\right) \cdot\left(x_{1}^{-1}, \ldots, x_{i}^{-1}, \ldots, x_{k}^{-1}\right) \\
& \cdot\left(x_{1 \rho(y)}, \ldots, x_{i}, \ldots, x_{k \rho(y)}\right) \cdot \rho(y) \\
= & \left(y_{1}^{x_{1}} \cdot x_{1}^{-1} \cdot x_{1 \rho(y)}, \ldots, y_{i}^{x_{i}}, \ldots, y_{k}^{x_{k}} \cdot x_{k}^{-1} \cdot x_{k \rho(y)}\right) \cdot \rho(y) .
\end{aligned}
$$

Thus $\pi_{i}\left(y^{x}\right)=y_{i}^{x_{i}} \in P_{i}$, i.e. $x_{i}$ lies in $X_{i}$ and so $N_{\mathbf{S}}(Q)$ lies in $\mathbf{X}$.

Consider $\mathbf{X} P \leqslant L \imath K$. Since $Q_{1} \times \cdots \times Q_{k}=O_{p}(\mathbf{X}) \in \operatorname{Syl}_{p}(\mathbf{X})$, the group $\mathbf{X} P$ has a normal series

$$
1 \triangleleft Q_{1} \times \cdots \times Q_{k}=O_{p}(\mathbf{X}) \preccurlyeq \mathbf{X} \preccurlyeq \mathbf{X} P .
$$

By Lemma 2.6, $\overline{N_{\mathbf{X}}(Q)}=N_{\overline{\mathbf{X}}}(\bar{Q})=C_{\overline{\mathbf{X}}}(\bar{Q})$, where

$$
-: P \mathbf{X} \rightarrow \overline{P \mathbf{X}}=P \mathbf{X} /\left(Q_{1} \times \cdots \times Q_{k}\right)
$$

is the natural homomorphism.

Now consider the corresponding natural homomorphisms

$$
\text { - }\left(X_{i} P_{i}\right) \rightarrow \overline{X_{i} P_{i}}=\left(X_{i} P_{i}\right) / Q_{i}
$$

(we simply restrict the above homomorphism on $X_{i} P_{i}$ ) for $i=1, \ldots, k$. Then each element $x=\left(x_{1}, \ldots, x_{k}\right) \in \mathbf{X}$ is mapped to $\bar{x}=\left(\bar{x}_{1}, \ldots, \bar{x}_{k}\right)$ and each element $y=\left(y_{1}, \ldots, y_{k}\right) \rho(y) \in Q$ is mapped to $\left(\bar{y}_{1}, \ldots, \bar{y}_{k}\right) \rho(y)$. Since $X_{i}$ normalizes $P_{i}$, we obtain that $\bar{X}_{i}$ centralizes $\overline{P_{i}}$, i.e. $\left(\bar{x}_{1}, \ldots, \bar{x}_{k}\right)$ centralizes $\left(\bar{y}_{1}, \ldots, \bar{y}_{k}\right)$. So

$$
\begin{aligned}
& \bar{x}^{\bar{y}}=\left(\bar{x}_{1}, \ldots, \bar{x}_{k}\right)^{\left(\bar{y}_{1}, \ldots, \bar{y}_{k}\right) \rho(y)}=\left(\bar{x}_{1}^{\bar{y}_{1}}, \ldots, \bar{x}_{k}^{\bar{y}_{k}}\right)^{\rho(y)} \\
& =\left(\bar{x}_{1}, \ldots, \bar{x}_{k}\right)^{\rho(y)}=\left(\bar{x}_{1 \rho(y)^{-1}}, \ldots, \bar{x}_{k \rho(y)^{-1}}\right) .
\end{aligned}
$$

Since $\rho(Q)=K \leqslant \operatorname{Sym}_{k}$ is transitive, we obtain that

$$
C_{\overline{\mathbf{X}}}(\bar{Q})=C_{\overline{\mathbf{X}}}(\rho(Q))=\left\{\left(\bar{x}_{1}, \ldots, \bar{x}_{k}\right) \mid \bar{x}_{1}=\cdots=\bar{x}_{k}\right\} .
$$

Thus

$$
\left|N_{\mathbf{S}}(Q)\right|_{p^{\prime}}=\left|\overline{N_{\mathbf{X}}(Q)}\right|=C_{\bar{X}}(\bar{Q})=\left|\overline{X_{1}}\right|=\left|X_{1}\right|_{p^{\prime}}=\left|N_{S_{1}}\left(P_{1}\right)\right|_{p^{\prime}}
$$

and (2.2) holds. 
Lemma 2.9. Assume $T$ is a normal subgroup of $G$ such that $T=G_{1} \times \cdots \times G_{k}$, and $G / T$ is a p-group. Assume also that $G$ acts on $\left\{G_{1}, \ldots, G_{k}\right\}$ by conjugation, i.e. for every $x \in G$ and $i=1, \ldots, k$ there exists $j \in\{1, \ldots, k\}$ with $G_{i}^{x}=G_{j}$. Denote by $\pi_{i}$ the natural projection $\pi_{i}: T \rightarrow G_{i}$. Let $H$ be a subgroup of $G$ such that for every $i=1, \ldots, k$ we have that $\pi_{i}(H \cap T)$ equals either $G_{i}$ or 1 , that is, $H \cap T$ is a subdirect product of some of $G_{i}$ 's. Then $v_{p}(H)$ divides $v_{p}(G)$.

Proof. Suppose that this lemma is false and consider a counterexample $(G, H)$ with $|G|$ minimal. Then $H \leqslant G$ is such that $H \cap T$ is a subdirect product of some of $G_{i}$ 's and $v_{p}(H)$ does not divide $v_{p}(G)$.

Let $\Omega_{1}, \ldots, \Omega_{s}$ be $G$-orbits of $\left\{G_{1}, \ldots, G_{k}\right\}$ and let $G_{i_{j}}$ be a fixed element of $\Omega_{j}$. In order to simplify notation, we assume $G_{i_{1}}=G_{1}$. Assume that $L_{1}$ is a minimal characteristic subgroup of $G_{1}$, then $L_{1}$ is a direct product of isomorphic simple groups, and either $L_{1}$ is elementary abelian or $L_{1}$ is a direct product of isomorphic nonabelian simple groups. For every $i=2, \ldots, s$, fix the corresponding characteristic subgroup $L_{i_{j}}$ of $G_{i_{j}}$ so that $L_{1} \simeq L_{i_{j}}$ and $G_{1} / L_{1} \simeq G_{i_{j}} / L_{i_{j}}$. Denote by $L$ the normal closure of $\left\langle L_{1}, \ldots, L_{i_{s}}\right\rangle$ in $G$, that is,

$$
L=\left\langle L_{i_{j}}^{x} \mid x \in G, j=1, \ldots, s\right\rangle .
$$

Evidently $L$ is a direct product of some groups isomorphic to $L_{1}$. In particular, $L$ is abelian if and only if $L_{1}$ is abelian and $L$ is a direct product of isomorphic nonabelian simple groups if and only if $L_{1}$ is a direct product of isomorphic nonabelian simple groups. Since $G$ acts transitively by conjugation on each $G$-orbit, we obtain that $L=\pi_{1}(L) \times \cdots \times \pi_{k}(L), \pi_{1}(L) \simeq \cdots \simeq \pi_{k}(L)$, and $G$ also acts by conjugation on $\left\{\pi_{1}(L), \ldots, \pi_{k}(L)\right\}$. Hence, if we consider the natural homomorphism

$$
\text { - } G \rightarrow \bar{G}=G / L,
$$

then $\bar{T}=\bar{G}_{1} \times \cdots \times \bar{G}_{k}=\left(G_{1} / \pi_{1}(L)\right) \times \cdots \times\left(G_{k} / \pi_{k}(L)\right)$. Clearly $\bar{G}$ acts by conjugation on $\left\{\bar{G}_{1}, \ldots, \bar{G}_{k}\right\}$ and $\bar{G} / \bar{T} \simeq G / T$ is a $p$-group. This shows that $\bar{G}$ satisfies the conditions of the lemma. We also have

$$
\pi_{i}(\bar{H} \cap \bar{T})=\pi_{i}(\overline{H L \cap T}),
$$

so $\pi_{i}(\bar{H} \cap \bar{T})=\overline{G_{i}}$ if $\pi(H \cap T)=G_{i}$ and $\pi_{i}(\bar{H} \cap \bar{T})=1$ if $\pi_{i}(H \cap T)=1$. Thus $\bar{H}$ also satisfies the conditions of the lemma. By induction, $v_{p}(\bar{H})$ divides $v_{p}(\bar{G})$.

Let $Q$ be a Sylow $p$-subgroup of $H$ and let $P$ be a Sylow $p$-subgroup of $G$ containing $Q$. By Lemma 2.1,

$$
v_{p}(H)=v_{p}(Q(H \cap L)) \cdot v_{p}(H /(H \cap L))=v_{p}(Q(H \cap L)) \cdot v_{p}(\bar{H})
$$


and

$$
v_{p}(G)=v_{p}(P L) \cdot v_{p}(G / L)=v_{p}(P L) \cdot v_{p}(\bar{G}) .
$$

Thus we need to show that $v_{p}(Q(H \cap L))$ divides $v_{p}(P L)$ in order to obtain a contradiction with the choice of $(G, H)$.

Assume first that $L$ is abelian. Then $P L$ is solvable, hence $v_{p}(Q(H \cap L))$ divides $v_{p}(P L)$ by Lemma 2.3 . Thus we may assume that $G_{1}$ has no characteristic abelian subgroups and so $G_{1}$ has no normal abelian subgroups.

Now assume that $L$ is nonabelian. As we note above, $L_{1}$ is a direct product of isomorphic nonabelian simple groups. So $L$ is also a direct product of isomorphic nonabelian simple groups, say $L=S_{1} \times \cdots \times S_{m}$. Then $G$ acts by conjugation on $\left\{S_{1}, \ldots, S_{m}\right\}$. Denote by $\psi_{j}: L \rightarrow S_{j}$ the natural projection. If $\pi_{i}(H \cap T)=1$, then clearly $\psi_{j}(H \cap L)=1$ for every $S_{j} \leqslant G_{i}$. Assume that $\pi_{i}(H \cap T)=G_{i}$. Since $H \cap L \S H$, we obtain that $\pi_{i}(H \cap L)$ is a normal subgroup of $\pi_{i}(H \cap T)=G_{i}$. Moreover, $\pi_{i}(H \cap L)$ is a normal subgroup of $\pi_{i}(L)=\prod_{S_{j} \leqslant L_{i}} S_{j}$, so $\pi_{i}(H \cap L)$ is a product of some of $S_{j}$ 's. Therefore, $H \cap L$ is a subdirect product of $S_{j}$ 's. This shows that $(P L, Q(H \cap L))$ satisfies the conditions of the theorem. If $P L \neq G$, then by induction $v_{p}(Q(H \cap L))$ divides $v_{p}(P L)$. Thus we may assume that $P L=G$.

Assume that $L$ is not a minimal normal subgroup in $P L$. Then there exists $K \triangleq P L$ such that $K<L$. Since $L=S_{1} \times \cdots \times S_{m}$ is a direct product of isomorphic simple groups, we obtain $K=S_{i_{1}} \times \cdots \times S_{i_{t}}$ for some $\left\{i_{1}, \ldots, i_{t}\right\} \subseteq$ $\{1, \ldots, m\}$. Consider a natural homomorphism $\varphi: P L \rightarrow P L / K$. Clearly $(\varphi(P L), \varphi(Q(H \cap L)))$ satisfies the conditions of the lemma, so by induction $v_{p}(\varphi(Q(H \cap L)))$ divides $v_{p}(\varphi(P L))$. It is also clear that $(P K, Q(H \cap K))$ also satisfies the conditions of the lemma, so by induction $v_{p}(Q(H \cap K))$ divides $v_{p}(P K)$. By Lemma 2.1,

$$
v_{p}(Q(H \cap L))=v_{p}(\varphi(Q(H \cap L))) \cdot v_{p}(Q(H \cap K))
$$

and

$$
v_{p}(P L)=v_{p}(\varphi(P L)) \cdot v_{p}(P K),
$$

so $v_{p}(Q(H \cap L))$ divides $v_{p}(P L)$. Hence, we may assume that $L$ is a minimal normal subgroup of $P L$.

Now we have that $G=P L$, where $P \in \operatorname{Syl}_{p}(G), L=S_{1} \times \cdots \times S_{m}$ is a minimal normal subgroup of $G$, and $S_{1}, \ldots, S_{m}$ are isomorphic (even $G$-conjugate) nonabelian simple groups. By Lemma 2.7, there exists an embedding $\varphi: G \rightarrow$ $\operatorname{Aut}_{G}\left(S_{1}\right)<\rho(G)$, where $\rho: G \rightarrow \operatorname{Sym}_{m}$ is the permutation representation corresponding to the action of $G$ on $\left\{S_{1}, \ldots, S_{m}\right\}$ by conjugation. Moreover, since $G=P L, \varphi$ can be chosen so that for every $P \leqslant X \leqslant G$ we have

$$
\varphi(X) \leqslant \operatorname{Aut}_{X}\left(S_{1}\right)<\rho(X)=\operatorname{Aut}_{X}\left(S_{1}\right)<\rho(G) .
$$


We show that $\varphi(G)$ as a subgroup of $\operatorname{Aut}_{G}\left(S_{1}\right) \prec \rho(G)$ satisfies the conditions of Lemma 2.8 .

Since $S_{1} \times \cdots \times S_{m} \leqslant \operatorname{Ker} \rho$, we obtain that $\rho(G)=\rho(P)$ is a transitive $p$-subgroup of $\operatorname{Sym}_{m}$. Now $S_{2} \times \cdots \times S_{m}$ centralizes $S_{1}$, so $\operatorname{Aut}_{G}\left(S_{1}\right) / S_{1}$ is a $p$-group. Thus $\operatorname{Aut}_{G}\left(S_{1}\right)$ with normal subgroup $S_{1}$ plays the role of the group $L$ with the notation of Lemma 2.8, while $\rho(G)$ is the group $K$ with the notation of Lemma 2.8.

Since $\varphi\left(S_{1} \times \cdots \times S_{m}\right)=S_{1} \times \cdots \times S_{m}$, we obtain that $S_{1} \times \cdots \times S_{m} \leqslant \varphi(G)$ and condition (a) of Lemma 2.8 is satisfied. Clearly

$$
\varphi(G)\left(\operatorname{Aut}_{G}\left(S_{1}\right) \times \cdots \times \operatorname{Aut}_{G}\left(S_{m}\right)\right)=\operatorname{Aut}_{G}\left(S_{1}\right) \imath \rho(G),
$$

so condition (b) of Lemma 2.8 is satisfied. By definition, $\pi_{i}\left(N_{G}\left(S_{i}\right)\right)=\operatorname{Aut}_{G}\left(S_{i}\right)$, thus condition (c) is true. Finally, by (2.3) we have

$$
\left.\varphi(P) \leqslant \operatorname{Aut}_{P}\left(S_{1}\right) \times \cdots \times \operatorname{Aut}_{P}\left(S_{m}\right)\right) \rtimes \rho(P)
$$

and by definition $\operatorname{Aut}_{P}\left(S_{i}\right)=\pi_{i}\left(N_{P}\left(S_{i}\right)\right)$, so condition (d) is also true. Thus

$$
v_{p}(G)=\left|S_{1}\right|_{p^{\prime}}^{m-1} \cdot v_{p}\left(\operatorname{Aut}_{G}\left(S_{1}\right)\right) \text {. }
$$

On the other hand, by Lemma 2.1,

$$
\begin{aligned}
v_{p}(P L) & =\left|L: N_{L}(P)\right|, \\
v_{p}(Q(H \cap L)) & =\left|(H \cap L): N_{H \cap L}(Q)\right| .
\end{aligned}
$$

Assume that $H \cap L \neq L$. Since $L$ is a direct product of nonabelian simple groups and $H \cap L$ is a subdirect product of some simple factors of $L$, we obtain that $|H \cap L|=\left|S_{1}\right|^{s}$ for some $s<m$. Clearly $\left|(H \cap L): N_{H \cap L}(Q)\right|$ divides $\left|S_{1}\right|_{p^{\prime}}^{s}$, since $\left|S_{1}\right|_{p^{\prime}}^{s}$ is the index of a Sylow $p$-subgroup of $H \cap L$. Since $s \leqslant m-1$, equation (2.4) implies that $v_{p}(H)$ divides $v_{p}(G)$.

Assume finally that $H \cap L=L$. Then from $Q \leqslant P$ we obtain $Q \cap L=$ $P \cap L \in \operatorname{Syl}_{p}(L)$. Hence the group $P N_{L}(P \cap L)$ has the normal series

$$
1 \triangleleft(P \cap L) \triangleleft N_{L}(P \cap L) \triangleleft P N_{L}(P \cap L),
$$

where $P \cap L$ is a $p$-group, $N_{L}(P \cap L) /(P \cap L)$ is a $p^{\prime}$-group, and

$$
P N_{L}(P \cap L) / N_{L}(P \cap L) \simeq P /\left(P \cap N_{L}(P \cap L)\right)
$$

is a $p$-group. By Lemma 2.6,

$$
N_{N_{L}(P \cap L)}(Q) \geqslant N_{N_{L}(P \cap L)}(P) .
$$

But, clearly, $N_{H \cap L}(Q)=N_{L}(Q)$ normalizes $Q \cap L$, so lies in $N_{L}(P \cap L)$. Hence

$$
N_{H \cap L}(Q)=N_{L}(Q)=N_{N_{L}(P \cap L)}(Q) \geqslant N_{N_{L}(P \cap L)}(P)=N_{L}(P),
$$

and so $v_{p}(Q(H \cap L))$ divides $v_{p}(P L)$. 


\section{Proof of the main theorem}

Assume Theorem 1.1 is false and let $G$ be a counterexample of minimal order. We proceed in a series of steps to get a contradiction.

Step 1. If $1 \neq A \preccurlyeq G$, then $G / A$ satisfies $\operatorname{DivSyl}(p)$.

Indeed, by Lemma 2.5, the group of induced automorphisms does not depend on the choice of an $(r c)$-series. Hence we may assume that

$$
1=G_{0}<G_{1}<\cdots<G_{n}=G
$$

goes through $A$, i.e. $A=G_{i}$ for some $i$. Consider a natural homomorphism

$$
-: G \rightarrow \bar{G}=G / A \text {. }
$$

Clearly

$$
\overline{1}=\bar{G}_{i}<\bar{G}_{i+1}<\cdots<\bar{G}_{n}=\bar{G}
$$

is an $(r c)$-series of $\bar{G}$. By [8, Lemma 1.2],

$$
\operatorname{Aut}_{\bar{G}}\left(\bar{G}_{j} / \bar{G}_{j-1}\right) \simeq \operatorname{Aut}_{G}\left(G_{j} / G_{j-1}\right)
$$

for $j=i+1, \ldots, n$, so $\bar{G}$ satisfies $\operatorname{DivSyl}(p)$ by minimal counterexample.

Step 2. The solvable radical $S(G)$ (that is the largest solvable normal subgroup of $G$ ) is trivial.

Suppose this is false. Then $G / S(G)$ satisfies DivSyl $(p)$ by Step 1. Moreover, for given $P \in \operatorname{Syl}_{p}(G)$, the group $P S(G)$ satisfies $\operatorname{DivSyl}(p)$ by Lemma 2.3. Hence $G$ satisfies $\operatorname{DivSyl}(p)$ by Lemma 2.2, a contradiction.

Step 3. Let $T=S_{1} \times \cdots \times S_{k}$ be a minimal normal subgroup of $G$. Then $G / T$ is a $p$-group. Consequently, $T$ is the unique minimal normal subgroup of $G$, and $S_{i}$ is a nonabelian simple group for $i=1, \ldots, k$.

Indeed, let $P$ be a Sylow $p$-subgroup of $G$. If $G / T$ is not a $p$-group, then $P T$ is a proper subgroup of $G$. By Lemma 2.5, we may assume that

$$
1=G_{0}<G_{1}<\cdots<G_{n}=G
$$

goes through $T$, that is, $T=G_{k}$ for some $k \in\{1, \ldots, n-1\}$. Denote the image of $P \cap N_{G}\left(G_{i} / G_{i-1}\right)$ in $\operatorname{Aut}_{G}\left(G_{i} / G_{i-1}\right)$ by $P_{i}$ for $i=1, \ldots, k$. By definition, $P_{i}\left(G_{i} / G_{i-1}\right)=\operatorname{Aut}_{P T}\left(G_{i} / G_{i-1}\right) \leqslant \operatorname{Aut}_{G}\left(G_{i} / G_{i-1}\right)$, and the conditions of the theorem say that for every $p$-subgroup $Q$ of $P_{i}\left(G_{i} / G_{i-1}\right)$ the group $Q\left(G_{i} / G_{i-1}\right)$ satisfies $\operatorname{DivSyl}(p)$. Hence $P T$ satisfies the conditions of the theorem, so by minimality, $P T$ satisfies $\operatorname{DivSyl}(p)$. Then Step 1 and Lemma 2.2 imply that $G$ satisfies $\operatorname{DivSyl}(p)$, a contradiction. 
Step 4. If $H$ is a subgroup of $G$ containing $T$, then $v_{p}(H)$ divides $v_{p}(G)$.

Assume that $T \leqslant H$. Let $Q$ be a Sylow $p$-subgroup of $H$. Since $G / T$ is a $p$-group, so is $H / T$, in particular, $H=Q T$. Let $P \in \operatorname{Syl}_{p}(G)$ be chosen so that $P \cap H=Q$. Then $P \cap T=Q \cap T \in \operatorname{Syl}_{p}(T)$ is a normal subgroup of $P$ and of $Q$. Denote $N_{T}(P \cap T)$ by $K$. Since $P \cap T \in \operatorname{Syl}_{p}(T)$, we have $P \cap T \in$ $\operatorname{Syl}_{p}(K)$, so $K /(P \cap T)$ is a $p^{\prime}$-group, and $P K$ satisfies the conditions of Lemma 2.6. Clearly both $N_{T}(Q)$ and $N_{T}(P)$ lie in $K$, so $N_{T}(Q)=N_{K}(Q)$ and $N_{T}(P)=N_{K}(P)$. By Lemma 2.6, $N_{T}(Q)=N_{K}(Q) \geqslant N_{K}(P)=N_{T}(P)$. Finally, by Lemma 2.1 ,

$$
\begin{aligned}
& v_{p}(H)=v_{p}(Q T)=\left|T: N_{T}(Q)\right|, \\
& v_{p}(G)=v_{p}(P T)=\left|T: N_{T}(P)\right| .
\end{aligned}
$$

Thus $v_{p}(H)$ divides $v_{p}(G)$.

Denote $\operatorname{Aut}_{G}\left(S_{i}\right)$ by $L_{i}$. Since $T$ is a unique minimal normal subgroup and $T$ is nonabelian, by Lemma 2.7, there exists an injective homomorphism $\varphi: G \rightarrow$ $L_{1} \prec \rho(G)$, where $\rho: G \rightarrow \operatorname{Sym}_{k}$ is the permutation representation corresponding to the action of $G$ on $\left\{S_{1}, \ldots, S_{k}\right\}$ by conjugation. Moreover, since $S_{i}$ is a unique minimal normal subgroup of $L_{i}$ and $\rho(G)$ is transitive, we have $\mathbf{S}=S_{1} \times \cdots \times S_{k}$ is a unique minimal normal subgroup of $L \imath \rho(G)$. In particular, for every such inclusion $\varphi$ the identity $\varphi(T)=\mathbf{S}$ holds. Below we identify $G$ with its image $\varphi(G) \leqslant L_{1} 2 \rho(G)$ and $T$ with $\mathbf{S}$. For every $H \leqslant G$ denote $\pi_{i}(H \cap T)$ by $H_{i}$, where $\pi_{i}$ is defined before Lemma 2.7. Clearly $H \cap T$ is a subdirect product of $H_{1}, \ldots, H_{k}$.

Step 5. If $H \leqslant G$ is chosen so that $H T=G$, then $\left|H_{1}\right|=\cdots=\left|H_{k}\right|$ and $H$ normalizes $H_{1} \times \cdots \times H_{k}$.

Since $T$ is a minimal normal subgroup of $G$ and $T$ is nonabelian, $G$ acts transitively by conjugation on $\left\{S_{1}, \ldots, S_{k}\right\}$. Since $H T=G$ and $T \leqslant \operatorname{Ker}(\rho)$, we obtain $\rho(H)=\rho(G)$, in particular, for every $i=1, \ldots, k$, there exists $x_{i} \in H$ such that $S_{1}^{x_{i}}=S_{i}$.

Let $h \in H \cap T$. Then we have $h=h_{1} \cdot \ldots \cdot h_{k}$, where $h_{i}=\pi_{i}(h) \in L_{i}$. Since $H \cap T \preccurlyeq H$, it follows that $h^{x_{i}} \in H \cap T$ for $i=1, \ldots, k$. On the other hand, $h^{x_{i}}=h_{1}^{x_{i}} \cdot \ldots \cdot h_{k}^{x_{i}}$ and this identity with the fact that $x_{i}$ permutes $S_{i}$ 's implies $\pi_{i}\left(h^{x_{i}}\right)=h_{1}^{x_{i}}$. Whence $H_{1}^{x_{i}} \subseteq H_{i}$. The same arguments show that

$$
H_{i}^{x_{i}^{-1}} \subseteq H_{1}
$$

Hence $H_{1}^{x_{i}}=H_{i}$ and $\left|H_{1}\right|=\left|H_{i}\right|$ for $i=1, \ldots, k$. These arguments also show that an arbitrary $x \in H$ permutes $H_{i}$ 's, so $x$ normalizes $H_{1} \times \cdots \times H_{k}$. 
Step 6. Assume $H \leqslant G$ is chosen so that $H T=G$.

- If $H \cap T=H_{1} \times \cdots \times H_{k}$, then $v_{p}(H)=v_{p}\left(\operatorname{Aut}_{H}\left(S_{1}\right)\right) \cdot\left|H_{1}\right|_{p^{\prime}}^{k-1}$ divides $v_{p}(G)$.

- If $H \cap T \neq H_{1} \times \cdots \times H_{k}$, then $v_{p}(H)$ divides $v_{p}\left(\operatorname{Aut}_{H}\left(S_{1}\right)\right) \cdot\left|H_{1}\right|_{p^{\prime}}^{k-1}$, and so $v_{p}(H)$ also divides $v_{p}(G)$.

Let $Q$ be a Sylow $p$-subgroup of $H$ and let $P \in \operatorname{Syl}_{p}(G)$ be chosen so that $P \cap H=Q$. By Step 3, $G / T$ is a $p$-group. So $H=Q(H \cap T)$ and $G=Q T$. By Lemma 2.7, there exists an embedding $\varphi: G \rightarrow \operatorname{Aut}_{G}\left(S_{1}\right) \gtrless \rho(G)$ such that for every $Q \leqslant X \leqslant G$ we have $\varphi(X) \leqslant \operatorname{Aut}_{X}\left(S_{1}\right)$ 2 $\rho(Q)$, so we choose the embed$\operatorname{ding} \varphi$ with this property. Clearly $G$ as a subgroup of $\operatorname{Aut}_{G}\left(S_{1}\right)<\rho(G)$ with Sylow $p$-subgroup $P \leqslant \operatorname{Aut}_{P}\left(S_{1}\right) \succ \rho(G)$ satisfies the conditions of Lemma 2.8, so $v_{p}(G)=\left|S_{1}\right|_{p^{\prime}}^{k-1} \cdot v_{p}\left(\operatorname{Aut}_{G}\left(S_{1}\right)\right)$. If $H \cap T=H_{1} \times \cdots \times H_{k}$, then $H$ as a subgroup of $\operatorname{Aut}_{H}\left(S_{1}\right) \geq \rho(H)$ with Sylow $p$-subgroup $Q \leqslant \operatorname{Aut}_{Q}\left(S_{1}\right) \gtrless \rho(G)$ satisfies the conditions of Lemma 2.8, so $v_{p}(H)=\left|H_{1}\right|_{p^{\prime}}^{k-1} \cdot v_{p}\left(\operatorname{Aut}_{H}\left(S_{1}\right)\right)$. Clearly $\left|\operatorname{Aut}_{H}\left(S_{1}\right)\right|$ divides $\left|\operatorname{Aut}_{G}\left(S_{1}\right)\right|$, while $v_{p}\left(\operatorname{Aut}_{H}\left(S_{1}\right)\right)$ divides $v_{p}\left(\operatorname{Aut}_{G}\left(S_{1}\right)\right)$ by the conditions of the theorem. Hence $v_{p}(H)$ divides $v_{p}(G)$.

If $H \cap T \neq H_{1} \times \cdots \times H_{k}$, then $H$ as a subgroup of $H\left(H_{1} \times \cdots \times H_{k}\right)$ satisfies the conditions of Lemma 2.9. So $v_{p}(H)$ divides $v_{p}\left(H\left(H_{1} \times \cdots \times H_{k}\right)\right)$, and $v_{p}\left(H\left(H_{1} \times \cdots \times H_{k}\right)\right)=\left|H_{1}\right|_{p^{\prime}}^{k-1} \cdot v_{p}\left(\operatorname{Aut}_{H}\left(S_{1}\right)\right)$ divides $v_{p}(G)$ in view of the previous paragraph. Hence $v_{p}(H)$ divides $v_{p}(G)$.

Step 7. If $H \leqslant G$ and $H T \neq G$, then $v_{p}(H)$ divides $v_{p}(G)$.

Since the nonabelian composition factors of $H T$ and $G$ are the same, like in the proof of Step 3, we can show that $H T$ satisfies the conditions of the theorem, so $H T$ satisfies $\operatorname{DivSyl}(p)$ by induction. Therefore $v_{p}(H)$ divides $v_{p}(H T)$. But by Step $4, v_{p}(H T)$ divides $v_{p}(G)$. Consequently, $v_{p}(H)$ divides $v_{p}(G)$.

In view of Steps 4, 6 and 7, we obtain that in any case $v_{p}(H)$ divides $v_{p}(G)$, which contradicts the choice of $G$. This completes the proof of Theorem 1.1.

\section{Remarks and questions}

In this section we discuss natural questions arising in connection with the main theorem. We now can prove the following result.

Proposition 4.1. Let $G$ be an almost simple group with simple socle $S$ such that $p$ does not divide $|S|$. Then $G$ satisfies $\operatorname{DivSyl}(p)$. 
Proof. By Corollary 2.4, we only need to verify that $P S$ satisfies $\operatorname{DivSyl}(p)$ for $P \in \operatorname{Syl}_{p}(G)$. Let $H$ be a subgroup of $P S$. We may assume that $P \cap H$ is a Sylow $p$-subgroup of $H$. Since $p$ does not divide $|S|$, we have $P S=S \rtimes P$, and so $H=(H \cap S) \rtimes(H \cap P)$. The known properties of coprime action (see, e.g., [4, Exercise 3E.4]) imply that

$$
v_{p}((H \cap S) \rtimes(H \cap P))=\left|(H \cap S): C_{H \cap S}(H \cap P)\right|
$$

divides the index $\left|S: C_{S}(H \cap P)\right|$, while $\left|S: C_{S}(H \cap P)\right|$ clearly divides $\left|S: C_{S}(P)\right|=v_{p}(P S)$. Therefore $v_{p}(H)$ divides $v_{p}(P S)$.

From Proposition 4.1 we immediately get the following remark.

Remark 4.2. The main theorem is a direct generalization of Navarro's theorem since all nonabelian composition factors of a $p$-solvable group are $p^{\prime}$-groups.

Remark 4.3. In the conditions of the main theorem, we assume that $P\left(G_{i} / G_{i-1}\right)$ satisfies $\operatorname{DivSyl}(p)$ for every $p$-subgroup $P$ of $\operatorname{Aut}_{G}\left(G_{i} / G_{i-1}\right)$. Corollary $2.4 \mathrm{im}$ plies that under the conditions of the main theorem every group $L$ with

$$
G_{i} / G_{i-1} \leqslant L \leqslant \operatorname{Aut}_{G}\left(G_{i} / G_{i-1}\right)
$$

satisfies $\operatorname{DivSyl}(p)$. However the authors do not know any example of an almost simple group $L$ with simple socle $S$ such that $L$ satisfies $\operatorname{DivSyl}(p)$, while $S$ does not. So it is natural to assert the following:

Conjecture 4.4. Let $S$ be a simple group satisfying DivSyl $(p)$. Then every $L$ such that $S \leqslant L \leqslant \operatorname{Aut}(S)$ satisfies $\operatorname{DivSyl}(p)$.

Remark 4.5. The structure of the normalizers of Sylow 2-subgroups in finite simple groups (see [5]) implies that in (infinitely) many almost simple groups the Sylow 2-subgroups are self-normalizing, so these groups satisfy DivSyl(2). Also in [12] the complete description of simple groups $\operatorname{PSL}_{2}(q)$ satisfying $\operatorname{DivSyl}(p)$ for every prime $p$ is obtained. In particular, in [12] an infinite series of simple groups, satisfying $\operatorname{DivSyl}(p)$ for every prime $p$ is obtained, so, if a group satisfies $\operatorname{DivSyl}(p)$ for every prime $p$, then it is not necessarily solvable.

Of course, other examples exist. We provide these examples just to show that there are many almost simple groups satisfying $\operatorname{DivSyl}(p)$ that are not $p^{\prime}$-groups.

In connection with Conjecture 4.4 and Remark 4.5, the following questions arise naturally: 
Problem 4.6. How often do almost simple groups satisfy DivSyl $(p)$ ?

Problem 4.7. What almost simple groups satisfy $\operatorname{DivSyl}(p)$ for every prime $p$ ?

Remark 4.8. Other possible questions arise if we try to consider $\pi$-Hall subgroups instead of Sylow $p$-subgroups. In [7], A. Turull proved that if $G$ is $\pi$-separable, then $v_{\pi}(H)$ divides $v_{\pi}(G)$ for every $H \leqslant G$. It is not hard to see that Lemmas 2.1-2.3 hold if we consider $\pi$-separable groups and $\pi$-Hall subgroups, so an alternative proof of Turull's theorem can be also obtained by the same arguments. But in order to extend Turull's theorem, one needs to introduce an appropriate class of finite groups so that each proper subgroup satisfies $E_{\pi}$ at least. We do not go into details in this case, we only mention that such a possibility exists and could be considered later.

Acknowledgments. The authors thank the anonymous referee for valuable suggestions and improvements of the text.

\section{Bibliography}

[1] F. Gross, On the existence of Hall subgroups, J. Algebra 98 (1986), no. 1, 1-13.

[2] M. Hall, Jr., On the number of Sylow subgroups in a finite group, J. Algebra 7 (1967), 363-371.

[3] B. Huppert, Endliche Gruppen. I, Grundlehren Math. Wiss. 134, Springer, Berlin, 1967.

[4] I. M. Isaacs, Finite Group Theory, Grad. Stud. Math. 92, American Mathematical Society, Providence, 2008.

[5] A. S. Kondrat'ev, Normalizers of Sylow 2-subgroups in finite simple groups, Math. Notes 78 (2005), no. 3-4, 338-346.

[6] G. Navarro, Number of Sylow subgroups in $p$-solvable groups, Proc. Amer. Math. Soc. 131 (2003), no. 10, 3019-3020.

[7] A. Turull, The number of Hall $\pi$-subgroups of a $\pi$-separable group, Proc. Amer. Math. Soc. 132 (2004), no. 9, 2563-2565.

[8] E. P. Vdovin, Carter subgroups in finite almost simple groups, Algebra Logika 46 (2007), no. 2, 157-216.

[9] E. P. Vdovin, Groups of induced automorphisms and their application for the study of the existence of Hall subgroups, Algebra Logika 53 (2014), no. 5, 643-648.

[10] E. P. Vdovin and V.I. Zenkov, The intersection of soluble Hall subgroups in finite groups, Tr. Inst. Mat. Mech. UrO RAN 15 (2009), no. 2, 74-83. 
[11] V. A. Vedernikov, Finite groups with Hall $\pi$-subgroups, Mat. Sb. 203 (2012), no. 3, 23-48.

[12] Z. Wu, W. Guo and E. P. Vdovin, On the number of Sylow subgroups in special linear groups of degree 2, Algebra Logic 56 (2017), no. 6, 498-501.

Received August 15, 2017; revised March 18, 2018.

\section{Author information}

Wenbin Guo, Department of Mathematics, University of Science and Technology of China, Hefei 230026, P. R. China.

E-mail: wbguo@ustc.edu.cn

Evgeny P. Vdovin, Departement of Mechanics and Mathematics, Novosibirsk State University; and Sobolev Institute of Mathematics, Novosibirsk 630090, Russia.

E-mail: vdovin@math.nsc.ru 